\title{
On the Solution of Differential Equations by Definite Integrals
}

\author{
By Professor E. T. WhitTakeR, F.R.S.
}

(Received 12th December 1930. Read 16th January 1931.)

\section{§1. Introduction.}

It is well known that in many cases the solutions of a linear differential equation can be expressed as definite integrals, different solutions of the same equation being represented by integrals which have the same integrand, but different paths of integration. Thus, the various solutions of the hypergeometric differential equation

$$
z(z-1) \frac{d^{2} y}{d z^{2}}+\{-c+(a+b+1) z\} \frac{d y}{d z}+a b y=0
$$

can be represented by integrals of the type

$$
\int t^{a-c}(t-1)^{c-b-1}(t-z)^{-a} d t
$$

the path of integration being (for one particular solution) a closed circuit encircling the point $t=0$ in the positive direction, then the point $t=1$ in the positive direction, then the point $t=0$ in the negative direction, and lastly the point $t=1$ in the negative direction; or (for another particular solution) an arc in the $t$-plane joining the points $t=1$ and $t=\infty$.

The object of the present paper is to communicate a general theorem regarding the solution of both ordinary and partial differential equations by means of families of definite integrals. The theorem gives only the integrand of the integral: but when in any particular case the integrand is known, there is usually not much difficulty in determining a double-circuit or other path of integration which will give any desired solution.

In $\S 2$ the theorem is stated in its general form. The rest of the paper is devoted to working out particular cases of it, the results being believed to be in most cases new.

\section{$\S 2$. The general theorem.}

The general theorem may be stated as follows:Consider any contact-transformation from a set of variables $\left(q_{1}, q_{2}, \ldots q_{n}\right.$, $\left.p_{1}, p_{2}, \ldots p_{n}\right)$ to a set of variables $\left(Q_{1}, Q_{2}, \ldots Q_{n}, P_{1}, P_{2}, \ldots P_{.}\right) . \quad$ As is 
known, ${ }^{1}$ we can obtain a contact-transformation by taking any function $W\left(q_{1}, q_{2}, \ldots q_{n}, Q_{1}, Q_{2}, \ldots Q_{n}\right)$ of the $q$ 's and $Q$ 's, and writing down the equations

$$
P_{r}=\frac{\partial W}{\partial Q_{r}}, \quad p_{r}=-\frac{\partial W}{\partial q_{r}} \quad(r=1,2, \ldots n)
$$

and solving them with respect to the $Q$ 's and $P$ 's. Suppose that the variables $\left(Q_{1}, Q_{2}, \ldots Q_{n}, P_{1}, P_{2}, \ldots P_{n}\right)$, when expressed in terms of $\left(q_{1}, q_{2}, \ldots q_{n}, p_{1}, p_{2}, \ldots p_{n}\right)$, are denoted by $Q_{r}\left(q_{1}, q_{2}, \ldots q_{n}, p_{1}, p_{2} \ldots p_{n}\right)$, $P_{r}\left(q_{1}, q_{2}, \ldots q_{n}, p_{1}, p_{2}, \ldots p_{n}\right) \quad(r=1,2, \ldots n)$. Then the set of partial differential equations

$$
\left.\begin{array}{l}
Q_{r}\left(q_{1}, q_{2}, \ldots q_{n}, \frac{\partial}{\partial q_{1}}, \frac{\partial}{\partial q_{2}}, \ldots \frac{\partial}{\partial q_{n}}\right) \chi=t_{r} \chi \\
P_{r}\left(q_{1}, q_{2}, \ldots q_{n}, \frac{\partial}{\partial q_{1}}, \frac{\partial}{\partial q_{2}}, \ldots \frac{\partial}{\partial q_{n}}\right) \chi=-\frac{\partial \chi}{\partial t_{r}}
\end{array}\right\}(r=1,2, \ldots n)
$$

is a compatible set of differential equations, and possesses a solution $\chi\left(q_{1}, q_{2}, \ldots q_{n}, t_{1}, t_{2}, \ldots t_{n}\right)$. It should be observed here that the equations (2) are symbolic: that is to say, $\left(\frac{\partial}{\partial q_{r}}\right)^{2} \chi$ is to be taken to mean $\left(\frac{\partial^{2} \chi}{\partial q_{r}^{2}}\right)$, etc.: and it should also be observed that certain rules are to be observed in replacing the $p$ 's by the $\frac{\partial}{\partial q}$ 's in $Q_{r}$ and $P_{r}$, in order to settle e.g. whether a term $p_{1} q_{1}$ is to become $q_{1} \frac{\partial}{\partial q_{1}}$ or $\frac{\partial}{\partial q_{1}} \cdot q_{1}$ : but this matter we defer for the present.

Now let it be required to solve a set of $n$ compatible linear partial differential equations in $n$ independent variables, say

$$
F_{r}\left(q_{1}, q_{2}, \ldots q_{n}, \frac{\partial}{\partial q_{1}}, \frac{\partial}{\partial q_{2}}, \ldots \frac{\partial}{\partial q_{n}}\right) \psi=0, \quad(r=1,2, \ldots n)
$$

where $\psi\left(q_{1}, q_{2}, \ldots q_{n}\right)$ is the function to be determined. Suppose that when the $q$ 's and $p$ 's are replaced by their values in terms of the $Q$ 's and $P$ 's from (1), the function $F_{r}\left(q_{1}, q_{2}, \ldots q_{n}, p_{1}, p_{2}, \ldots p_{n}\right)$ becomes $G_{r}\left(Q_{1}, Q_{2}, \ldots Q_{n}\right.$, $\left.P_{1}, P_{2}, \ldots P_{n}\right)$. Then the solution of the partial differential equations (3) is furnished by a family of definite integrals of the type

$\psi\left(q_{1}, q_{2}, \ldots q_{n}\right)=\iiint \ldots \int x\left(q_{1}, q_{2}, \ldots q_{n}, t_{1}, t_{2}, \ldots t_{n}\right) \phi\left(t_{1}, t_{2}, \ldots t_{n}\right) d t_{1} d t_{2} . . d t_{n}$

I Cf. e.g. Whittaker, Analytical Dynamics, §126. 
when $\phi\left(t_{1}, t_{2}, \ldots t_{n}\right)$ is the solution of the set of $n$ compatible linear partial differential equations

$$
G_{r}\left(t_{1}, t_{2}, \ldots t_{n} \frac{\partial}{\partial t_{1}}, \frac{\partial}{\partial t_{2}}, \ldots \frac{\partial}{\partial t_{n}}\right) \phi=0 \quad(r=1,2, \ldots n) .
$$

My own original way of establishing the theorem was defective as a proof, and has been superseded by a proof devised by Dr Kermack and $\mathrm{Dr} \mathrm{M}^{\circ} \mathrm{Crea}$, which is given in the paper following this. I will therefore not discuss the proof here, but confine myself to working out particular cases, chiefly with a view to obtaining new results.

§3. An extension of Laplace's transformation.

As a first example, let us suppose that the contact-transformation is specified by the equations

when

$$
P_{r}=\frac{\partial W}{\partial Q_{r}}, \quad p_{r}=-\frac{\partial W}{\partial q_{r}} \quad(r=1,2, \ldots n)
$$

so that

$$
W=-q_{1} Q_{1}-q_{2} Q_{2}-\ldots-q_{n} Q_{n},
$$

$$
P_{r}=-q_{r}, \quad p_{r}=Q_{r} \quad(r=1,2, \ldots n)
$$

The equations (2) for the function $\chi$ become

$$
\frac{\partial \chi}{\partial q_{r}}=t_{r} \chi, \quad \frac{\partial \chi}{\partial t_{r}}=q_{r} \chi \quad(r=1,2 \ldots n)
$$

of which the solution is

$$
\chi=e^{q_{1} t_{1}+q_{2} t_{2}+. .+q_{n} t_{n}}
$$

(neglecting now, as always, an arbitrary multiplicative constant). Thus the theorem asserts that if $\psi\left(q_{1}, q_{2}, \ldots q_{n}\right)$ is a solution of $n$ compatible partial differential equations

$$
F_{r}\left(q_{1}, q_{2}, \ldots q_{r}, \frac{\partial}{\partial q_{1}}, \frac{\partial}{\partial q_{2}}, \ldots \frac{\partial}{\partial q_{n}}\right) \psi=0, \quad(r=1,2, \ldots n)
$$

then a relation exists of the type

$\psi\left(q_{1}, q_{2}, \ldots q_{n}\right)=\iint \ldots \int e^{q_{1} t_{1}+q_{2} t_{2}+\ldots .+\eta_{n} t_{n}} \phi\left(t_{1}, t_{2}, \ldots t_{n}\right) d t_{1} d t_{2} . . d t_{n}$

where $\phi\left(t_{1}, t_{2}, \ldots t_{n}\right)$ is a solution of the set of compatible partial differential equations

$$
F_{r}\left(-\frac{\partial}{\partial t_{1}},-\frac{\partial}{\partial t_{2}}, \ldots-\frac{\partial}{\partial t_{n}}, t_{1}, t_{2}, \ldots t_{n}\right) \phi=0 .
$$


It will be seen that this is essentially the extension, to partial differential equations in $n$ variables, of the well-known method, ${ }^{1}$ due to Laplace, for solving ordinary linear differential equations by means of integrals of the form

$$
\int e^{q t} \phi(t) d t
$$

As a particular case, suppose that the equations (5) are

$$
\left.\begin{array}{c}
\left(\frac{\partial}{\partial q_{1}}-a_{11} q_{1}-a_{12} q_{2}-\ldots-a_{1 n} q_{n}\right) \psi=0 \\
\left(\frac{\partial}{\partial q_{2}}-a_{21} q_{1}-a_{22} q_{2}-\ldots \ldots-a_{2 n} q_{n}\right) \psi=0 \\
\ldots \ldots \ldots \ldots \ldots \ldots \ldots \ldots \ldots \ldots \ldots \ldots \ldots \ldots \ldots \\
\left(\frac{\partial}{\partial q_{n}}-a_{n 1} q_{1}-a_{n 2} q_{2}-\ldots \ldots a_{n}\right) \psi=0
\end{array}\right\}
$$

where the $a^{\prime}$ s are constants, and $a_{i k}=a_{k i}$. These partial differential equations are compatible, the solution being evidently

$$
\psi\left(q_{1}, q_{2}, \ldots q_{n}\right)=e^{\frac{1}{2}\left(a_{11} q_{1}{ }^{2}+a_{2: 2} q_{2}^{2}+. .+a_{n n} q_{n}^{2}+2 a_{12} q_{1} q_{2}+. .\right)} .
$$

The corresponding equations (7) for $\phi$ are

$$
\left.\begin{array}{c}
\left(t_{1}+a_{11} \frac{\partial}{\partial t_{1}}+a_{12} \frac{\partial}{\partial t_{2}}+\ldots \ldots+a_{1 n} \frac{\partial}{\partial t_{n}}\right) \phi=0 \\
\left(t_{2}+a_{21} \frac{\partial}{\partial t_{1}}+a_{22} \frac{\partial}{\partial t_{2}}+\ldots \ldots+a_{2 n} \frac{\partial}{\partial t_{n}}\right) \phi=0 \\
\ldots \ldots \ldots \ldots \ldots \ldots \ldots \ldots \ldots \ldots \ldots \ldots \ldots \\
\left(t_{n}+a_{n 1} \frac{\partial}{\partial t_{1}}+a_{n 2} \frac{\partial}{\partial t_{2}}+\ldots \ldots+a_{n n} \frac{\partial}{\partial t_{n}}\right) \phi=0
\end{array}\right\}
$$

If we denote the determinant $\left\|a_{i k}\right\|$ by $\Delta$, and denote $(1 / \Delta) \times$ the co-factor of $a_{i k}$ in $\Delta$ by $A_{i k}$, so that the quadratic form $\sum_{i k} A_{i k} x_{i} x_{k}$ is the reciprocal of the quadratic form $\sum_{i k} a_{i k} x_{i} x_{k}$, then the last set of equations may be written

$$
\left.\begin{array}{l}
\left(\frac{\partial}{\partial t_{1}}+A_{11} t_{1}+A_{12} t_{2}+\ldots+A_{1 n} t_{n}\right) \phi=0 \\
\left(\frac{\partial}{\partial t_{2}}+A_{21} t_{1}+A_{22} t_{2}+\ldots \ldots+A_{2 n} t_{n}\right) \phi=0 \\
\cdots \ldots \ldots \ldots \ldots \ldots \ldots \ldots \ldots \ldots \ldots \\
\left(\frac{\partial}{\partial t_{n}}+A_{n 1} t_{1}+A_{n 2} t_{2}+\ldots \ldots+A_{n n} t_{n 2}\right) \phi=0
\end{array}\right\}
$$

I Of., e.g., A. R. Forsyth, Treatise on Differential Equations, Chapter VII., "Solution by Definite Integrals." 
and they are compatible, their solution being

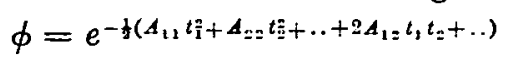

Thus the theorem asserts that a relation exists of the form

$$
\begin{array}{r}
\exp \left(\frac{1}{2} \sum_{i, k} a_{i k} q_{i} q_{k}\right)=\mathrm{constant} \times \iint \\
\exp \left(-\frac{1}{2} \sum_{i, k} A_{i k} t_{i} t_{k}\right) d t_{1} d t_{2} \ldots d t_{n},
\end{array}
$$

where the paths of integration may consist of loops surrounding the singularities of the integrand, or paths to infinity. Since the integrand has no finite singularities, we infer that the paths must terminate at infinity: and in fact we readily find that the precise equation is

$$
\begin{aligned}
\exp \left(\frac{1}{2} \sum_{i, k} a_{i k} q_{i} q_{k}\right)=(2 \pi)^{-\frac{1}{2} n} \Delta^{-\frac{1}{2}} \int_{-\infty}^{\infty} \int_{-\infty}^{\infty} & \int_{-\infty}^{\infty} \exp \left(q_{1} t_{1}+q_{2} t_{2}+\ldots\right. \\
& \left.+q_{n} t_{n}-\frac{1}{2} \sum_{i, k} A_{i k} t_{i} t_{k}\right) d t_{1} d t_{2} \ldots d t_{n},
\end{aligned}
$$

which represents the result of the theorem in this particular case. We can represent the expression $\sum_{i, k} A_{i k} t_{i} t_{k}$ in terms of the $a$ 's in the form

$$
-\frac{1}{\Delta}\left|\begin{array}{ccccc}
a_{11} & a_{12} & . . & a_{1 n} & t_{1} \\
a_{21} & a_{22} & . . & a_{2 n} & t_{2} \\
. . & . . & . . & . . & . . \\
. & . . & . & . & . . \\
a_{n 1} & a_{n 2} & . . & a_{2 n} & t_{n} \\
t_{1} & t_{2} & . . & t_{n} & 0
\end{array}\right| .
$$

§4. A double-integral connected with the hypergeometic function.

As a second example, suppose that the contact-transformation is specified by the function

so that

$$
W=-q_{1} \log Q_{1}-q_{2} \log Q_{2},
$$

$$
\begin{array}{llll}
q_{1}=-Q_{1} P_{1}, & q_{2}=-Q_{2} P_{2}, & p_{1}=\log Q_{1}, & p_{2}=\log Q_{2}, \\
\text { or } \quad Q_{1}=e^{p_{1}} & Q_{2}=e^{p_{2}}, & P_{1}=-e^{-p_{1}} q_{1}, & P_{2}=-e^{-p_{2}} q_{2} .
\end{array}
$$

The equations (2) for determining $\chi$ are now

$$
\begin{gathered}
\left\{\exp \left(\frac{\partial}{\partial q_{1}}\right)\right\} \chi=t_{1} \chi, \quad \text { or } \chi\left(q_{1}+1, q_{2}\right)=t_{1} \chi\left(q_{1}, q_{2}\right) \\
\left\{\exp \left(\frac{\partial}{\partial q_{2}}\right)\right\} \chi=t_{2} \chi, \quad \text { or } \chi\left(q_{1}, q_{2}+1\right)=t_{2} \chi\left(q_{1}, q_{2}\right) \\
-\left\{\exp \left(-\frac{\partial}{\partial q_{1}}\right)\right\} q_{1} \chi=-\frac{\partial \chi}{\partial t_{1}}, \quad \text { or }\left(q_{1}-1\right) \chi\left(q_{1}-1, q_{2}\right)=\frac{\partial \chi\left(q_{1}, q_{2}\right)}{\partial t_{1}} \\
-\left\{\exp \left(-\frac{\partial}{\partial q_{2}}\right)\right\} q_{2} \chi=-\frac{\partial \chi}{\partial t_{2}}, \quad \text { or }\left(q_{2}-1\right) \chi\left(q_{1}, q_{2}-1\right)=\frac{\partial \chi\left(q_{1}, q_{2}\right)}{\partial t_{2}}
\end{gathered}
$$

and the solution of these equations is

$$
\chi=t_{1}^{q_{1}-1} t_{2}^{q_{2}-1} \text {. }
$$


Thus the theorem asserts that the solution of a pair of compatible partial differential equations

$$
\left\{\begin{array}{l}
F_{1}\left(q_{1}, q_{2}, \frac{\partial}{\partial q_{1}}, \frac{\partial}{\partial q_{2}}\right) \psi=0 \\
F_{2}\left(q_{1}, q_{2}, \frac{\partial}{\partial q_{1}}, \frac{\partial}{\partial q_{2}}\right) \psi=0
\end{array}\right.
$$

may be expressed by a definite integral

$$
\psi\left(q_{1}, q_{2}\right)=\iint \phi\left(t_{1}, t_{2}\right) t_{1}^{q_{1}-1} t_{2}^{q_{2}-1} d t_{1} d t_{2},
$$

where $\phi$ is a solution of the equations

$$
\left.\begin{array}{l}
F_{1}\left(-t_{1} \frac{\partial}{\partial t_{1}},-t_{2} \frac{\partial}{\partial t_{2}}, \log t_{1}, \log t_{2}\right) \phi=0 \\
F_{2}\left(-t_{1} \frac{\partial}{\partial t_{1}},-t_{2} \frac{\partial}{\partial t_{2}}, \log t_{1}, \log t_{2}\right) \phi=0 .
\end{array}\right\}
$$

As a particular case, take

$$
\psi\left(q_{1}, q_{2}\right)=\Gamma\left(q_{1}\right) \Gamma\left(q_{2}\right) F\left(q_{1}, q_{2}, \frac{1}{2}, z\right),
$$

where $F(a, b, c, z)$ denotes the hypergeometric function. From the recurrence-formulae for the hypergeometric function we see that $\psi$ satisfies the two difference-equations

$$
\begin{aligned}
\psi\left(q_{1}, q_{2}+1\right)-\psi( & \left(q_{1}+1, q_{2}\right)+\left(q_{1}-q_{2}\right) \psi\left(q_{1}, q_{2}\right)=0 \\
\left\{\frac{1}{2}-2 q_{1}-\left(q_{2}-q_{1}\right) z\right\} & \psi\left(q_{1}, q_{2}\right)+(1-z) \psi\left(q_{1}+1, q_{2}\right) \\
-\left(\frac{1}{2}-q_{1}\right)\left(q_{1}-1\right) \psi\left(q_{1}-1, q_{2}\right) & =0,
\end{aligned}
$$

which may be written

$$
\left\{\begin{array}{c}
\left(e^{\partial / \partial q_{2}}-e^{\partial / \partial q_{1}}+q_{1}-q_{2}\right) \psi=0 \\
\left\{\frac{1}{2}-2 q_{1}-\left(q_{2}-q_{1}\right) z+(1-z) e^{\partial / \partial q_{1}}-\left(\frac{1}{2}-q_{1}\right)\left(q_{1}-1\right) e^{-\partial / \partial q_{1}}\right\} \psi=0,
\end{array}\right.
$$

so the corresponding equations (9) for $\phi$ are

$$
\left\{\begin{array}{c}
\left(t_{2}-t_{1}-t_{1} \frac{\partial}{\partial t_{1}}+t_{2} \frac{\partial}{\partial t_{2}}\right) \phi=0 \\
\left\{\frac{1}{2}+2 t_{1} \frac{\partial}{\partial t_{1}}+z t_{2} \frac{\partial}{\partial t_{2}}-z t_{1} \frac{\partial}{\partial t_{1}}+(1-z) t_{1}+\left(\frac{1}{2}+t_{1} \frac{\partial}{\partial t_{1}}\right)\left(t_{1} \frac{\partial}{\partial t_{1}}+1\right)\left(\frac{1}{t_{1}}\right)\right\} \phi=0
\end{array}\right.
$$

The solution to equation (11) is

$$
\phi=e^{-t_{1}-t_{2}} f(s),
$$

where $s=t_{1} t_{2}$, and $f$ denotes an arbitrary function. 
Equation (12) may be written

$$
\frac{1}{2} \phi+2 t_{1} \frac{\partial \phi}{\partial t_{1}}+z t_{2} \frac{\partial \phi}{\partial t_{2}}-z t_{1} \frac{\partial \phi}{\partial t_{1}}+(1-z) t_{1} \phi+\frac{1}{2} \frac{\partial \phi}{\partial t_{1}}+t_{1} \frac{\partial^{2} \phi}{\partial t_{1}^{2}}=0 .
$$

Substituting in this the value of $\phi$ given by (13), it becomes

$$
s \frac{d^{2} f}{d s^{2}}+\frac{1}{2} \frac{d f}{d s}-z f=0,
$$

of which the solution is

$$
f=\cosh \left(2 \sqrt{z t_{1} t_{2}}+\epsilon\right),
$$

where $\epsilon$ denotes a constant. The theorem (8) therefore gives $\Gamma\left(q_{1}\right) \Gamma\left(q_{2}\right) \boldsymbol{F}\left(q_{1}, q_{2}, \frac{1}{2}, z\right)=$

$$
\text { Constant. } \iint e^{-t_{1}-t_{2}} \cosh \left(2 \sqrt{t_{1} t_{2} z}+\epsilon\right) t_{1}^{q_{1}-1} t_{2}^{q_{2}-1} d t_{1} d t_{2} ;
$$

it is not difficult to determine the paths of integration and the constants, the final result being

$\Gamma\left(q_{1}\right) \Gamma\left(q_{2}\right) F\left(q_{1}, q_{2}, \frac{1}{2}, z\right)=\int_{0}^{\infty} \int_{0}^{\infty} e^{-t_{1}-t_{2}} \cosh \left(2 \sqrt{t_{1} t_{2} z}\right) t_{1}^{q_{1}-1} t_{2}^{q_{2}-1} d t_{1} d t_{2}$.

§5. A new property of the hypergeometric function.

It is well-known' from the work of Pincherle and Mellin that the hypergeometric function can be represented by definite integrals in which the integrand is a product of Gamma-functions, e.g.

$$
\begin{array}{r}
F^{\prime}(\alpha, \beta, \gamma, 1-q)=\frac{1}{2 \pi i} \frac{\Gamma(\gamma)}{\Gamma(\alpha) \Gamma(\beta) \Gamma(\gamma-\beta) \Gamma(\gamma-\alpha)} \times \\
\int_{C} \Gamma(-t) \Gamma(a+t) \Gamma(\beta+t) \Gamma(\gamma-a-\beta-t) q^{t} d t,
\end{array}
$$

where the path of integration $C$ is parallel to the imaginary axis in the $t$-plane and passes between the sequences of positive and negative poles of the integrand. It will now be shown that these PincherleMellin integrals are particular cases of a much more general formula which expresses a new property of the hypergeometric function.

'Of. e.g. Whittaker and Watson, Modern Analysis, §14.5. 
For this purpose, we consider the contact-transformation defined by the function

so that the equations

$$
W=-Q \log (-q)
$$

$$
P=\frac{\partial W}{\partial Q}, \quad p=-\frac{\partial W}{\partial q},
$$

give

$$
Q=q p, \quad P=-\log (-q) .
$$

The equations (2) for $\chi$ are now

$$
q \frac{\partial \chi}{\partial q}=t \chi, \quad-\log (-q) \chi=-\frac{\partial \chi}{\partial t},
$$

giving

$$
\chi=(-q)^{t} \text {. }
$$

Thus the theorem (4) asserts that any linear differential equation

$$
F\left(q, \frac{d}{d q}\right) \psi=0
$$

is satisfied by a family of definite integrals of the type

$$
\psi(q)=\int(-q)^{t} \phi(t) d t
$$

where $\phi(t)$ is a solution of the differential equation

In particular, let

$$
F\left(-e^{-\frac{d}{d \bar{l}}},-e^{\frac{d}{d t}} \cdot t\right) \phi=0 .
$$

$$
\psi(q)=F(\alpha, \beta, \gamma, z+q),
$$

where $F$ now denotes the hypergeometric function, so that $\psi$ satisfies the differential equation (corresponding to (15) above)

$$
\left[\left\{(z+q) \frac{d}{d q}+a\right\}\left\{(z+q) \frac{d}{d q}+\beta\right\}-\left\{(z+q) \frac{d}{d q}+\gamma\right\} \frac{d}{d q}\right] \psi=0 .
$$

The equation for $\phi(t)$, corresponding to (17), is therefore

$$
\begin{aligned}
& \quad\left\{\left(-z e^{\frac{d}{d t} t}+t+a\right)\left(-z e^{\frac{d}{d t}} t+t+\beta\right)+\left(-z e^{\frac{d}{d z}} t+t+\gamma\right) e^{\frac{d}{a t}} t\right\} \phi=0 \\
& \text { or } \\
& z(z-1)(t+1)(t+2) \phi(t+2) \\
& +(t+1)\{\gamma+t-(\lambda+\beta+2 t+1) z\} \phi(t+1)+(a+t)(\beta+t) \phi(t)=0,
\end{aligned}
$$

a difference-equation whose solution is found (by comparing it with the recurrence-formulae for the hypergeometric function) to be

$$
\phi(t)=\frac{\Gamma(-t) \Gamma(\alpha+t) \Gamma(\beta+t)}{\Gamma(\gamma+t)} F(\alpha+t, \beta+t, \gamma+t, z) .
$$


Thus the equation (16) becomes $F(\alpha, \beta, \gamma, z+q)=$ Constant $\times$

$$
\int \frac{\Gamma(-t) \Gamma(\alpha+t) \Gamma(\beta+t)}{\Gamma(\gamma+t)} F(\alpha+t, \beta+t, \gamma+t, z)(-q)^{t} d t
$$

It is readily seen that the path of integration must be the same as for the Pincherle-Mellin integral (14), and in fact the complete formula is

$F(\alpha, \beta, \gamma, z+q)=\frac{1}{2 \pi i} \frac{\Gamma(\gamma)}{\Gamma(\alpha) \Gamma(\beta)} \times$

$\int_{c} \frac{\Gamma(-t) \Gamma(\alpha+t) \Gamma(\beta+t)}{\Gamma(\gamma+t)} F(\alpha+t, \beta+t, \gamma+t, z)(-q)^{t} d t$.

This formula expresses the general hypergeometric function of the sum of two arguments, $z$ and $q$, in terms of hypergeometric functions of the argument $z$. The Pincherle-Mellin formula (14) is evidently the special case of it which arises when $z=1$, so that the hypergeometric function in the integrand reduces to a product of Gamma-functions; but in this special case the most characteristic property of the more general formula (18) is lost.

§6. The linear differential equation with four regular singularities.

It is well-known ${ }^{1}$ that the linear differential equation of the second order with three regular singularities, that is, the equation of the general hypergeometric function or Riemann $P$-function

$$
P\left\{\begin{array}{llll}
a & b & c & \\
a & \beta & \gamma & z \\
a^{\prime} & \beta^{\prime} & \gamma^{\prime} &
\end{array}\right\}
$$

can be solved by means of definite integrals of the type

$$
\int(t-a)^{p}(t-b)^{q}(t-c)^{r}(z-t)^{v} d t .
$$

It is therefore natural to enquire whether the linear differential equation of the second order with four regular singularities, that is the equation of the function

$$
P\left\{\begin{array}{lllll}
a & b & c & e & \\
a & \beta & \gamma & \epsilon & z \\
a^{\prime} & \beta^{\prime} & \gamma^{\prime} & \epsilon^{\prime} &
\end{array}\right\}
$$

can be solved by means of definite integrals of the type

$$
\int(t-a)^{p}(t-b)^{2}(t-c)^{r}(t-e)^{s}(z-t)^{v} d t .
$$

1 Cf. e.f., Whittaker and Watson, Modern Analysis, § 14.6. 
The answer is in the negative: in fact definite integrals of this last type do occur ${ }^{1}$ in the solution of linear differential equations with four singularities, but these linear differential equations are of the third order.

To discuss this question, consider the contact-transformation specified by the function

so that

$$
W=-(n+1) \log (q-Q)
$$

$$
p=-\frac{\partial W}{\partial q}=\frac{n+\mathrm{I}}{q-Q} \quad, \quad P=\frac{\partial W}{\partial Q}=\frac{n+1}{q-Q},
$$

and therefore

$$
Q=q-\frac{n+1}{p} \quad, \quad P=p .
$$

The equations (2) for $\chi$ thus become

Now (19) may be written

$$
\begin{aligned}
\left\{q-(n+1)\left(\frac{\partial}{\partial q}\right)^{-1}\right\} \chi & =t_{\chi} \\
\frac{\partial \chi}{\partial q} & =-\frac{\partial \chi}{\partial t} .
\end{aligned}
$$

or

$$
\frac{\partial}{\partial q}\{(q-t) \chi\}=(n+1) \chi
$$

This with equation (20) gives

$$
\frac{1}{\chi} \frac{\partial \chi}{\partial q}=\frac{n}{q-t} .
$$

$$
\chi=(t-q)^{n} \text {. }
$$

Thus the theorem (4) asserts that a linear differential equation

$$
F\left(q, \frac{d}{d q}\right) \psi=0
$$

is satisfied by a family of integrals of the type

$$
\psi(q)=\int(t-q)^{n} \phi(t) d t
$$

where $\phi(t)$ is a solution of the differential equation

$$
F\left\{t+(n+1)\left(\frac{d}{d t}\right)^{-1}, \frac{d}{d t}\right\} \phi=0 .
$$

1 Such integrals have been used in the solution of third-order equations by $\mathrm{L}$. Schlesinger, Math. ZS., 27 (1928), p. 504. 
In particular, suppose that

$$
\phi(t)=t^{a}(t-1)^{\beta}(t-e)^{\gamma},
$$

which obviously satisfies the differential equation

$$
\left(\frac{d}{d t}-\frac{a}{t}-\frac{\beta}{t-1}-\frac{\gamma}{t-e}\right) \phi=0 .
$$

Now since when $c$ is any constant and $\phi$ is any function of $t$, we have

therefore

$$
\left\{\frac{d}{d t}(t-c)-1\right\} \phi=\left\{(t-c) \frac{d}{d t}\right\} \phi,
$$

$$
t-c=\left\{\frac{d}{d t}(t-c)-1\right\}\left(\frac{d}{d t}\right)^{-1}
$$

and therefore equation (21) may be written

$$
\begin{aligned}
{\left[\frac{d}{d t}-\frac{a}{\left\{\frac{d}{d t} t-1\right\}\left(\frac{d}{d t}\right)^{-1}}-\right.} & \frac{\beta}{\left\{\frac{d}{d t}(t-1)-1\right\}\left(\frac{d}{d t}\right)^{-1}} \\
& \left.-\frac{\gamma}{\left\{\frac{d}{d t}(t-e)-1\right\}\left(\frac{d}{d t}\right)^{-1}}\right] \phi=0 .
\end{aligned}
$$

or, since $\frac{1}{H K^{-1}} \phi=K H^{-1} \phi$, whatever operators $H$ and $K$ may be,

$$
\frac{d}{d t}\left[1-\frac{\alpha}{\frac{d}{d t} t-1}-\frac{\beta}{\frac{d}{d t}(t-1)-1}-\frac{\gamma}{\frac{d}{d t}(t-e)-1}\right] \phi=0
$$

or, operating on this with $\left(\frac{d}{d t}\right)^{-1}$

$$
\left[1-\frac{\alpha}{\frac{d}{d t} t-1}-\frac{\beta}{\frac{d}{d t}(t-1)-1}-\frac{\gamma}{\frac{d}{d t}(t-e)-1}\right] \phi=0 .
$$

Now the above theorem asserts that the integral

$$
\psi(q)=\int t^{a}(t-1)^{\beta}(t-e)^{\gamma}(t-q)^{n} d t
$$

satisfies the equation which is obtained from (22) by replacing $\frac{d}{d t}$ by $\frac{d}{d q}$ and replacing $t$ by $\left\{q-(n+1)\left(\frac{d}{d q}\right)^{-1}\right\}$. Thus the integral (23) satisfies the equation

$$
\left[1-\frac{a}{\frac{d}{d q} q-n-2}-\frac{\beta}{\frac{d}{d q}(q-1)-n-2}-\frac{\gamma}{\frac{d}{d q}(q-e)-n-2}\right] \psi=0 .
$$




$$
\begin{aligned}
& \text { Operating with } \frac{d}{d q}(q-e)-(n+2), \text { we obtain } \\
& {\left[\frac{d}{d q}(q-e)-n-2-\frac{a}{\left\{\frac{d}{d q} q-n-2\right\}\left\{\frac{d}{d q}(q-e)-n-2\right\}^{-1}}\right.} \\
& \left.-\frac{\beta}{\left\{\frac{d}{d q}(q-1)-n-2\right\}\left\{\frac{d}{d q}(q-e)-n-2\right\}^{-1}}-\gamma\right] \psi=0 .
\end{aligned}
$$

Now we have

$$
\left\{\frac{d}{d q}(q-1)-n-1\right\}\left\{\frac{d}{d q}(q-e)-n-2\right\}=\left\{\frac{d}{d q}(q-e)-n-1\right\}\left\{\frac{d}{d q}(q-1)-n-2\right\}
$$

and therefore

$$
\left\{\frac{d}{d q}(q-e)-n-1\right\}^{-1}\left\{\frac{d}{d q}(q-1)-n-1\right\}=\left\{\frac{d}{d q}(q-1)-n-2\right\}\left\{\frac{d}{d q}(q-e)-n-2\right\}^{-1} .
$$

Thus (24) may be written

$$
\begin{aligned}
{\left[\frac{d}{d q}(q-e)-n-2-\frac{a}{\left\{\frac{d}{d q}(q-e)-n-1\right\}^{-1}\left\{\frac{d}{d q} q-n-1\right\}}\right.} \\
\left.-\frac{\beta}{\left\{\frac{d}{d q}(q-e)-n-1\right\}^{-1}\left\{\frac{d}{d q}(q-1)-n-1\right\}}-\gamma\right] \psi=0
\end{aligned}
$$

or

$$
\begin{aligned}
{\left[\frac{d}{d q}(q-e)\right.} & -n-2-\left\{\frac{d}{d q} q-n-1\right\}^{-1}\left\{\frac{d}{d q}(q-e)-n-1\right\} a \\
& \left.-\left\{\frac{d}{d q}(q-1)-n-1\right\}^{-1}\left\{\frac{d}{d q}(q-e)-(n+1)\right\} \beta-\gamma\right] \psi=0 .
\end{aligned}
$$

Operating with $\frac{d}{d q}(q-1)-(n+1)$, we have

$$
\left[\begin{array}{c}
{\left[\frac{d}{d q}(q-1)-n-1\right\}\left\{\frac{d}{d q}(q-e)-n-2\right\}} \\
-\left\{\frac{d}{d q}(q-1)-n-1\right\}\left\{\frac{d}{d q} q-n-1\right\}^{-1}\left\{\frac{d}{d q}(q-e)-n-1\right\} \alpha \\
-\left\{\frac{d}{d q}(q-e)-n-1\right\} \beta-\left\{\frac{d}{d q}(q-1)-(n+1)\right\} \gamma
\end{array}\right] \psi=0,
$$


or, using a formula similar to (28),

$\left[\begin{array}{c}\left\{\frac{d}{d q}(q-1)-n-1\right\}\left\{\frac{d}{d q}(q-e)-n-2\right\} \\ -\left(\frac{d}{d q} q-n\right)^{-1}\left\{\frac{d}{d q}(q-1)-n\right\}\left\{\frac{d}{d q}(q-e)-n-1\right\} a \\ -\left\{\frac{d}{d q}(q-e)-n-1\right\} \beta-\left\{\frac{d}{d q}(q-1)-n-1\right\} \gamma\end{array}\right] \psi=0$,

or, operating with $\left(\frac{d}{d q} q-n\right)$,

$\left[\begin{array}{c}\left\{\frac{d}{d q} q-n\right\}\left\{\frac{d}{d q}(q-1)-n-1\right\}\left\{\frac{d}{d q}(q-e)-n-2\right\} \\ -\left\{\frac{d}{d q}(q-1)-n\right\}\left\{\frac{d}{d q}(q-e)-n-1\right\} a \\ -\left\{\frac{d}{d q}-n\right\}\left\{\frac{d}{d q}(q-e)-n-1\right\} \beta-\left\{\frac{d}{d q}-n\right\}\left\{\frac{d}{d q}(q-1)-n-1\right\} \gamma\end{array}\right] \psi=0$,

or, performing the operations,

$$
\begin{aligned}
& \frac{d^{3} \psi}{d q^{3}}+\left(\frac{1-a-n}{q}+\frac{1-\beta-n}{q-1}+\frac{1-\gamma-n}{q-e}\right) \frac{d^{2} \psi}{d q^{2}} \\
&+(n-1)\left\{\frac{a+\beta+n}{q(q-1)}+\frac{a+\gamma+n}{q(q-e)}+\frac{\beta+\gamma+n}{(q-1)(q-e)}\right) \frac{d \psi}{d q} \\
&+\frac{n(1-n)(\alpha+\beta+\gamma+n+1)}{q(q-1)(q-e)} \psi=0 .
\end{aligned}
$$

This is the differential equation satisfied by the family of definite integrals (23). It has:-

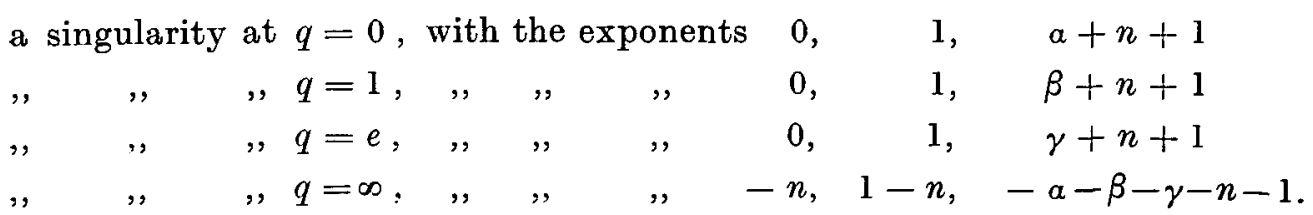

Thus the integral (23) satisfies a linear differential equation with four regular singularities, but this differential equation is of the third order. We might indeed have guessed that it could not in general satisfy a differential equation of the second order, since the number of independent functions which can be obtained from the integral (23), by taking different double-circuit paths of integration round the various singularities, is greater than the number of independent solutions of a linear differential equation of the second order. 
§7. Integrals involving hypergeometric functions.

We shall now consider the solution of linear differential equations by means of definite integrals which involve a hypergeometric function in the integrand.

By taking the function $W(q, Q)$ which determines the contacttransformation to be a function of the product $q Q$, we see that the equations

$$
Q=p \sigma(q p), \quad P=-q / \sigma(q p),
$$

where $\sigma(q p)$ is any function of the product $q p$, define a contacttransformation. Hence, in particular, the equations

$$
\left\{\begin{array}{l}
Q=1-\frac{(q p+\gamma) p}{(q p+\alpha)(q p+\beta)} \\
P=\frac{q(q p+\alpha)(q p+\beta)}{q p+\gamma}
\end{array}\right.
$$

define a contact-transformation. The corresponding equations (2) for $\chi(q, t)$ are

$$
\begin{aligned}
& \left\{1-\left(q \frac{\partial}{\partial q}+\beta\right)^{-1}\left(q \frac{\partial}{\partial q}+a\right)^{-1}\left(q \frac{\partial}{\partial q}+\gamma\right) \frac{\partial}{\partial q}\right\} \chi=t_{\chi}, \\
& q\left(q \frac{\partial}{\partial q}+\gamma\right)^{-1}\left(q \frac{\partial}{\partial q}+a\right)\left(q \frac{\partial}{\partial q}+\beta\right) \chi=-\frac{\partial \chi}{\partial t} .
\end{aligned}
$$

Now (26) gives

$$
\left(q \frac{\partial}{\partial q}+\beta\right)^{-1}\left(q \frac{\partial}{\partial q}+a\right)^{-1}\left(q \frac{\partial}{\partial q}+\gamma\right) \frac{\partial}{\partial q} \chi=(1-t) \chi
$$

or, operating with $q\left(q \frac{\partial}{\partial q}+\gamma\right)^{-1}\left(q \frac{\partial}{\partial q}+\alpha\right)\left(q \frac{\partial}{\partial q}+\beta\right)$ on both sides,

$$
\begin{aligned}
q \frac{\partial \chi}{\partial q} & =(1-t) q\left(q \frac{\partial}{\partial q}+\gamma\right)^{-1}\left(q \frac{\partial}{\partial q}+a\right)\left(q \frac{\partial}{\partial q}+\beta\right) \chi \\
& =-(1-t) \frac{\partial \chi}{\partial t}, \text { by (27). }
\end{aligned}
$$

Thus $\chi$ satisfies the partial differential equation

$$
q \frac{\partial \chi}{\partial q}+(1-t) \frac{\partial \chi}{\partial t}=0
$$

whose solution is $\chi=\theta(s)$, where $s=q-q t$ and $\theta$ is the arbitrary function. 
Substituting this value of $\chi$ in (27), we have

or

$$
\begin{aligned}
& \left(s \frac{d}{d s}+\gamma\right)^{-1}\left(s \frac{d}{d s}+a\right)\left(s \frac{d}{d s}+\beta\right) \theta=\frac{d \theta}{d s} \\
& \left(s \frac{d}{d s}+a\right)\left(s \frac{d}{d s}+\beta\right) \theta-\left(s \frac{d}{d s}+\gamma\right) \frac{d \theta}{d s}=0,
\end{aligned}
$$

which gives $\theta=F(a, \beta, \gamma, s)$, where $F$ is the hypergeometric function. Thus

$$
\chi=F(a, \beta, \gamma, q-q t) \text {, }
$$

and the theorem (4) asserts that if $\phi(t)$ is a solution of a linear differential equation

$$
G\left(t, \frac{d}{d t}\right) \phi=0
$$

then the linear differential equation

$$
\begin{aligned}
G\left\{1-\left(q \frac{d}{d q}+\beta\right)^{-1}\left(q \frac{d}{d q}+a\right)^{-1}\left(q \frac{d}{d q}+\gamma\right) \frac{d}{d q},\right. \\
\left.q\left(q \frac{d}{d q}+\gamma\right)^{-1}\left(q \frac{d}{d q}+a\right)\left(q \frac{d}{d q}+\beta\right)\right\} \psi=0
\end{aligned}
$$

is satisfied by a family of definite integrals of the type

$$
\psi(q)=\int F(a, \beta, \gamma, q-q t) \phi(t) d t .
$$

In particular, take $\phi(t)=t^{\kappa}$, so that the equation (28) becomes

$$
\left\{\frac{d}{d t} t-\kappa-1\right\} \phi=0
$$

and therefore the equation (29), satisfied by $\psi$, becomes

$$
\left\{q\left(q \frac{d}{d q}+\gamma\right)^{-1}\left(q \frac{d}{d q}+a\right)\left(q \frac{d}{d q}+\beta\right)-q \frac{d}{d q}-\kappa-1\right\} \psi=0,
$$

or $\left\{\left(q \frac{d}{d q}+a\right)\left(q \frac{d}{d q}+\beta\right)-\left(q \frac{d}{d q}+\gamma\right) \frac{d}{d q}-(\kappa+1)\left(q \frac{d}{d q}+\gamma\right)\left(\frac{1}{q}\right)\right\} \psi=0$

or $\quad q(q-1) \frac{d^{2} \psi}{d q^{2}}+\{(\alpha+\beta+1) q-(\gamma+\kappa+1)\} \frac{d \psi}{d q}+\left\{\alpha \beta-\frac{(\kappa+1)(\gamma-1)}{q}\right\} \psi=0$,

which is the differential equation of the function

$$
\psi=q^{-\kappa-1} F(\alpha-\kappa-1, \beta-\kappa-1, \gamma-\kappa-1, q),
$$

where $F$ denotes the hypergeometric function.

Thus the formula (30) becomes

$q^{-\kappa-1} F(a-\kappa-1, \beta-\kappa-1, \gamma-\kappa-1, q)=$ Constant $\times \int F(a, \beta, \gamma, q-q t) t^{\kappa} d t$ or, writing $t$ for $q t$, and $s$ for $-\kappa-1$, $F(\alpha+s, \beta+s, \gamma+s, q)=$ Constant $\times \int F(\alpha, \beta, \gamma, q-t) t^{-s-1} d t$. 
It is not difficult to determine the path of integration and the constant, the final result being $F(\alpha+s, \beta+s, \gamma+s, q)=\frac{\Gamma(\alpha) \Gamma(\beta) \Gamma(\gamma+s)}{\Gamma(\gamma) \Gamma(-s) \Gamma(a+s) \Gamma(\beta+s)} \int_{0}^{\infty} F(\alpha, \beta, \gamma, q-t) t^{-s-1} d t$.

This somewhat remarkable property of the hypergeometric function, which is new to me, is in a certain sense the reciprocal of the other new property expressed by equation (18), being in fact (as Dr Copson has kindly pointed out to me) connected with it by the Riemann-Mellin Theorem. ${ }^{1}$

\section{§8. Results involving Bessel functions.}

If the contact-transformation is taken to be

$$
Q=-p^{2}-\frac{p}{q}, \quad P=\frac{q}{2 p},
$$

the equations to determine $\chi$ are

$$
\left\{\begin{aligned}
\left(-\frac{\partial^{2}}{\partial q^{2}}-\frac{1}{q} \frac{\partial}{\partial q}\right) \chi & =t \chi \\
\frac{1}{2}\left(\frac{\partial}{\partial q}\right)^{-1} q \chi & =-\frac{\partial \chi}{\partial t},
\end{aligned}\right.
$$

and these have the solution $\chi=J_{0}(q \sqrt{ } t)$ where $J_{0}$ is the Bessel function of zero order: from this it is easy to derive such formulae as

$$
\exp \left(-\frac{q^{2}}{2 \kappa^{2}}\right)=\kappa^{2} \int_{0}^{\infty} \exp \left(-\frac{1}{2} \kappa^{2} t\right) J_{0}(q \sqrt{ } t) d t
$$

Similarly the contact-transformations

$$
Q=-p^{2}-\frac{p}{q}+\frac{n^{2}}{q^{2}}, \quad P=-\frac{1}{2} p q /\left(-p^{2}-\frac{p}{q}+\frac{n^{2}}{q^{2}}\right) .
$$

gives the $\chi$-function $\chi=J_{n}(q \sqrt{ } t)$ and leads to definite integrals having the Bessel function of order $n$ in the integrand.

1 Riemann, Berlin Monatsb., Nov. 1859: Mellin, Acta Math., 25 (1902), p. 138 : Math. Ann.,.68 (1909), p. 305. 